\title{
Simulation of Radiation Damage to Neural Cells with the Geant4-DNA Toolkit
}

\author{
Lkhagvaa Bayarchimeg $^{1, \star}$, Munkhbaatar Batmunkh ${ }^{1,2, \star \star}$, Oleg Belov ${ }^{1, \star \star \star}$, and \\ Oidov Lkhagva ${ }^{2, \star \star \star \star}$ \\ ${ }^{1}$ Laboratory of Radiation Biology, Joint Institute for Nuclear Research, 141980, Dubna \\ ${ }^{2}$ Division of Natural Sciences, National University of Mongolia, 210646, Ulaanbaatar
}

\begin{abstract}
To help in understanding the physical and biological mechanisms underlying effects of cosmic and therapeutic types of radiation on the central nervous system (CNS), we have developed an original neuron application based on the Geant 4 Monte Carlo simulation toolkit, in particular on its biophysical extension Geant4-DNA. The applied simulation technique provides a tool for the simulation of physical, physico-chemical and chemical processes (e.g. production of water radiolysis species in the vicinity of neurons) in realistic geometrical model of neural cells exposed to ionizing radiation. The present study evaluates the microscopic energy depositions and water radiolysis species yields within a detailed structure of a selected neuron taking into account its soma, dendrites, axon and spines following irradiation with carbon and iron ions.
\end{abstract}

\section{Introduction}

The investigation of radiation damage to the central nervous system (CNS) has been an on-going challenge for the last decades primarily due to the issues of brain radiotherapy and radiation protection for astronauts during space travel. Although recent findings revealed a number of molecular mechanisms associated with radiation-induced impairments in behaviour and cognition, some uncertainties exist in the initial neuronal cell injury leading to the further development of CNS malfunction. As usual, these initial stages of neuronal injury are hardly accessible to experimental measurements. Many events cannot be investigated experimentally at all. In this regard, development of computational methods for assessing these early stages of radiation damage to CNS is of great interest.

The irradiation with heavy charged particles is known to be a cause of numerous neurological disturbances in the CNS, leading to various behaviour and cognitive impairments [1]. The effects therefore cannot be completely understood without examining the earliest stages of the radiation injury to individual neurons. The initial radiation-induced events depend on the kinetic energy of the incident particles, and some of this energy can be transferred to the atoms and molecules during the incident particle pass through the target volume. The ionized or excited molecules produce new charged ions, free radicals and highly reactive chemical species that can break the chemical bonds and result thus

\footnotetext{
$\star$ e-mail: bayarchimeg@jinr.ru

$\star \star$ e-mail: batmunkh@jinr.ru

$\star \star \star$ e-mail: dem@jinr.ru

$\star \star \star \star$ e-mail: dorjtoinol@gmail.com
} 
in cells and tissues damage. Discrete Monte Carlo techniques are efficiently used to address this issue and to perform detailed modeling of the stochastic energy depositions within the trajectory of the incident particle and all secondary low energy particles. The neural cells have complex morphologies and narrow-tree-like structures with large area that makes them unique targets for particle irradiation.

The one of the most suitable tools for the simulation of the radiation transport inside and outside neural cells is the general-purpose Geant 4 Monte Carlo toolkit, which is used in a wide range of applications, including particle physics, accelerator design, space engineering, medical physics, education and industrial domains $[2,3]$. The Geant 4 extension to the very low energies encompasses the Geant4-DNA models serving to the calculation of initial radiation damage at the molecular level and showing good agreement with the experimental cross sections for nanodosimetry [4, 5].

We have extended a Geant4-based original application called NEURON to the investigation of the initial mechanisms of radiation-induced events in CNS, involving detailed modeling of the physicochemical processes in the vicinity of the neurons [6-8]. The purpose of the present work is to describe the capabilities of this radiobiology application for the simulation of single neuron irradiation with energetic heavy ions.

\section{The NEURON user application}

NEURON is a new radiobiology application of the Geant4 Monte Carlo simulation toolkit which reads any standardized neuromorphometric format (SWC) file representing the neuron morphology and estimates the microscopic energy depositions and production of water radiolysis species in the vicinity of the neural cells. The SWC file format is an output file defining the most realistic geometrical description of the neuron structure generated by digitally tracing tools based on 3D confocal microscopy images. Many laboratories are freely sharing their tracing reconstructions within SWC formats via online archives such as the NeuroMorpho.Org repository. A typical neuron morphology is composed of a cell body (soma), a single axon and dendritic trees with thousands of spines. These structures are simulated through combination of spherical and cylindrical volumes representing the smallest neuronal elements known as compartments (two interconnected morphological points). The source code of NEURON is written in $\mathrm{C}++$, where classes are defined which implement joining morphological points, geometrical transformations, rotations, calculation of the volume and the overall dimensions of the neuron, a bounding volume etc. Before computation, the user loads a standardized SWC file of a neuron and generates a bounding volume and a homogeneous spherical medium with dimensions automatically extrapolated using the SWC file describing 3D coordinates of a neuron morphology. The homogeneous medium contains both volumes of neural cell and bounding volume, that are all filled with liquid water. Geant 4 condensed electromagnetic models are used outside the neuron structure and Geant4-DNA discrete physicochemical models are activated inside the neuron structure, which is declared as a G4Region (Figure 1a). Nevertheless, the user can change the physical and chemical processes in the simulated media via simple UI commands, such as G4EmLivermorePhysics (condensed) or G4EmDNAPhysics (discrete) for all the medium, or a combination of condensed and discrete processes outside and inside the neuron structure. The primary particle is starting at a random position on the sphere surface (homogeneous medium) and uniformly directed towards the neuron structure (Figure 1b).

Neuronal morphological data of a single granule cell (GranuleCell-Nr2.CNG.swc file) from NeuroMorpho.Org has been selected as an example for our application which is found in the dentate gyrus region of mouse hippocampus (Figure 1). The overall dimensions (width $\times$ height $\times$ depth) of the selected neuron are calculated $\left(252 \times 317 \times 64-\mu \mathrm{m}^{3}\right)$ from coordinates of morphological points. The total volume and length of the selected neuron are $5048.43 \mu \mathrm{m}^{3}$ and $2191.54 \mu \mathrm{m}$, respectively. 

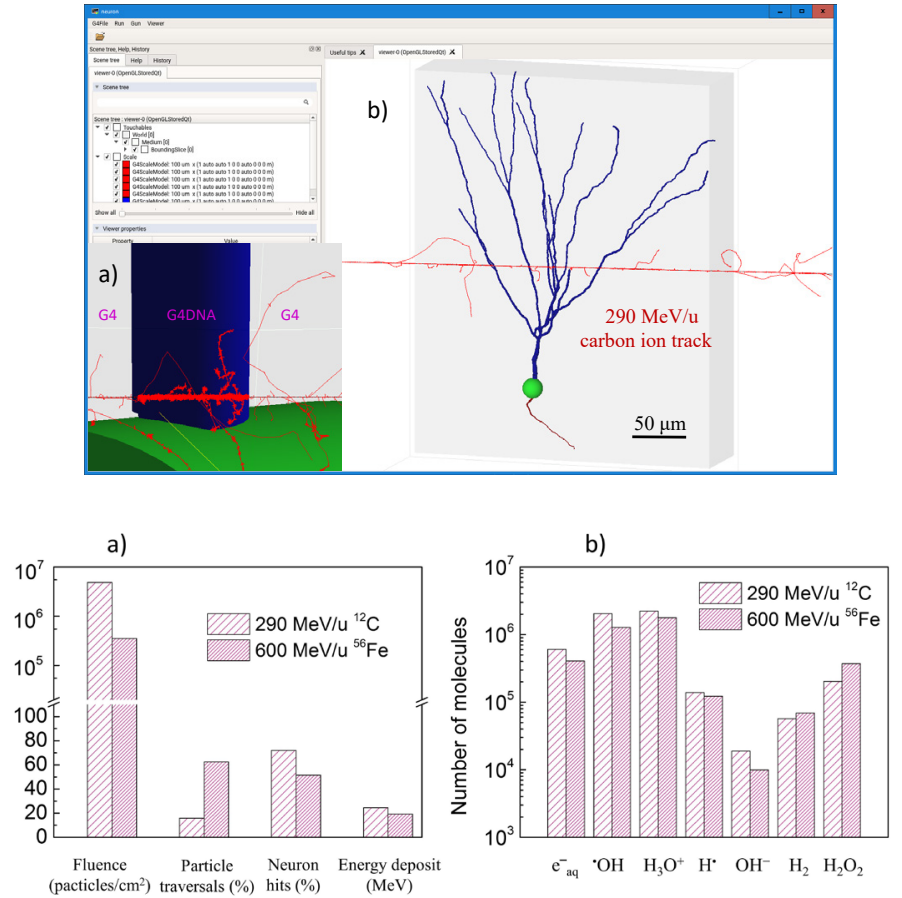

Figure 1. Screenshot of the NEURON application in the Geant 4 (version 10.2-patch02) environment with Qt GUI. (a) - demonstration of Geant4 and Geant4-DNA models of particle track passing through a compartment of neuron. (b) irradiation of a single granule cell with track structure of a carbon ion beam.

Figure 2. Summary of simulation results. (a) - particle fluence at the given dose, particle neuron traversal probability, hit percentage of neuron and total energy deposition. (b) - total number of water radiolysis species in neuron at $1 \mathrm{~ns}$ after exposure to 0.1 Gy carbon and iron ions.

\section{Calculation of microscopic energy depositions and production of water radiolysis species in the neuron model}

In the present study, a neuron is assumed to be exposed to $0.1 \mathrm{~Gy}$ carbon and iron ions with kinetic energies of $290 \mathrm{MeV} / \mathrm{u}$ and $600 \mathrm{MeV} / \mathrm{u}$, respectively. The fluence dose of $0.1 \mathrm{~Gy}$ is equivalent to 12809 carbon ions and 938 iron ions in the simulation medium. The simulation results are reported in Figure 2 where simulations of the direct and indirect effects on a single neuron under irradiation with carbon and iron ions with same fluence dose are reported.
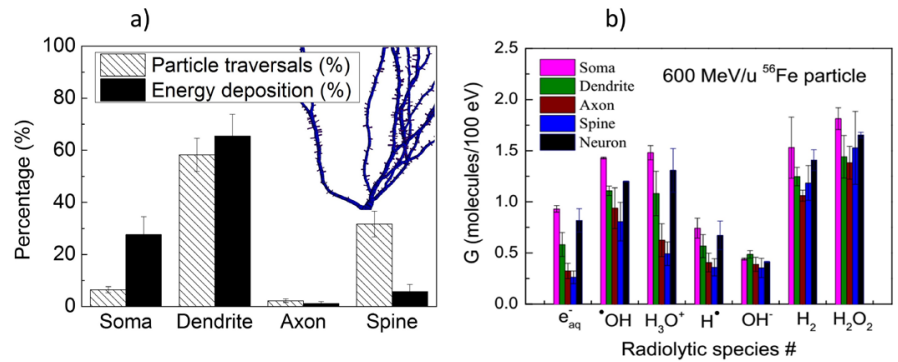

Figure 3. Analysis of energy depositions (a) and production of water radiolysis species (b) in single neuron's components, including soma, dendrite, axon and spines after irradiation with $600 \mathrm{MeV} / \mathrm{u}$ iron ions.

In the case of $0.1 \mathrm{~Gy}$ iron ions, particle traversals are longer than those of the carbon ions, however, the total number of neuron hits and the energy deposition are larger for the carbon ions as compared to the iron ions. The most widespread products of the water radiolysis are the solvated electrons $\mathrm{e}_{\mathrm{aq}}^{-}$, hydroxyl radical ${ }^{\bullet} \mathrm{OH}$, hydronium ion $\mathrm{H}_{3} \mathrm{O}^{+}$, hydrogen radical $\mathrm{H}^{\bullet}$, hydroxide ion $\mathrm{OH}^{-}$, dihydrogen $\mathrm{H}_{2}$, and hydrogen peroxide $\mathrm{H}_{2} \mathrm{O}_{2}$. Under exposure to iron ions, the total number of $\mathrm{H}_{2}$ and $\mathrm{H}_{2} \mathrm{O}_{2}$ species are larger than those of the carbon ions at a same given dose (Figure 2b). In the current 
study we have obtained morphological points of dendritic spines from original experimental data of selected neurons [9] that included coordinates and sizes of only spine heads, do not include neck information and spines morphology. The diameter and length of the spine neck were generated from ratio of spines head/neck diameter and total length derived from literature [10]. Thus, 2446 spines were simulated and each spine defined as two solids of sphere and cylinder, neck diameter depending on various size of spines head as ratio of 1.1. Figure 3 presents detailed results of the analysis of energy depositions and production of chemical species in the neutron structure, taking into account such cell components as soma, dendrites, axon and spines. In this case, most particles are traversing the structure of dendrites and spines. In addition, the energy deposition occurred mainly in dendrites rather than in other structures at the given dose of iron ions. As a result (Figure 3b), the hydrogen peroxide is the most observed product in soma and dendrites after irradiation.

\section{Conclusions}

The paper presented simulation results of the new NEURON application of the Geant4 and Geant4DNA toolkits. The applied simulation tool allows for the modeling of neuron cell irradiation, including physical, physico-chemical and chemical processes (e.g. production of water radiolysis species in the vicinity of the neurons). It uses realistic geometrical models of the neurons generated from a standardized SWC file representing the neuron morphology and a multi-scale discrete/non-discrete combination of Geant4-DNA and Geant4 electromagnetic processes. We have obtained accurate estimates for the probability of particle traversals, distribution of microscopic energy depositions, hit percentage of neuron structures and number of water radiolysis species in chosen neuron and such cell components as soma, dendrites, axon and spines after irradiation of energetic carbon and iron ions. In the case of carbon ions $(290 \mathrm{MeV} / \mathrm{u})$, the total energy deposition and number of most water radiolysis in a neuron were observed larger than those of the iron ions $(600 \mathrm{MeV} / \mathrm{u})$ at a same dose. The NEURON application helps to understand the initial physico-chemical events of ionizing radiation action in the CNS in the context of space radiobiology problems.

\section{Acknowledgements}

The study was conducted as part of the joint research protocol between the Laboratory of Radiation Biology of the Joint Institute for Nuclear Research (JINR) and the National University of Mongolia. Authors acknowledge the financial support from the JINR Theme 04-9-1077-2015/2017.

\section{References}

[1] A. G. Belyaeva et al., Neurochemical Journal 11, 2, 168-175 (2017)

[2] S. Agostinelli et al., Nucl Instrum Methods Phys Res Section A 506, 250-303 (2003)

[3] J. Allison et al., Nuclear Instruments and Methods A 835, 186-225, (2016)

[4] S. Incerti et al., Med. Phys. 37, 4692-4708 (2010)

[5] M. A. Bernal et al., Phys. Med. 31, 861-874 (2015)

[6] M. Batmunkh et al., J. Radiat. Res. Appl. Sci. 8, 498-507 (2015)

[7] O. V. Belov, M. Batmunkh, S. Incerti, and O. Lkhagva, Physica Medica 32, 1510-1520 (2016)

[8] M. Batmunkh, O.V. Belov, L. Bayarchimeg, and O. Lkhagva, Mong. J. Phys. 2, 317-323 (2016)

[9] M. Vuksic et al., HIPPOCAMPUS 18, 36, 4-375 (2008)

[10] A. Rodriguez et al., PLOS one 3(4), e1997 (2008) 